\title{
Hamiltonian systems with orbital, orbital inverse shadowing
}

Manseob Lee*

"Correspondence:

Imsds@mokwon.ac.kr

Department of Mathematics,

Mokwon University, Daejeon,

302-729, Korea

\begin{abstract}
We show that if a Hamiltonian system has the robustly orbitally shadowing property, then it is Anosov. Moreover, if a Hamiltonian system has the robustly orbitally inverse shadowing property with respect to the class of continuous methods, then it is Anosov.

MSC: 37C10; 37C50; 37D20

Keywords: Hamiltonian systems; Hamiltonian star systems; shadowing; orbital shadowing; inverse shadowing; orbital inverse shadowing; Anosov
\end{abstract}

\section{Introduction}

The various shadowing theory (shadowing, orbital shadowing, inverse shadowing, orbital inverse shadowing property) is close to the stability theory. In fact, Sakai has shown in [1] that if a diffeomorphism belongs to the $C^{1}$-interior of the set of all diffeomorphisms having the shadowing property, then it is a structurally stable diffeomorphism. Pilyugin $e t$ $a l$. [2] proved that if a diffeomorphism belongs to the set of all diffeomorphisms having the orbital shadowing property, then it is a structurally stable diffeomorphism. It extends the result of the shadowing property. For the orbital shadowing property, we can find many results (see [2-8]).

The notion of the inverse shadowing property is a dual notion of the shadowing property which was introduced by Corless and Pilyugin in [9]. Pilyugin [10] and Lee [11] proved that if a diffeomorphism belongs to the set of all diffeomorphisms having the inverse shadowing property with respect to the class the continuous method then it is a structurally stable diffeomorphism. The qualitative theory of dynamical systems with the property was developed by various researchers (see [7-18]). The inverse shadowing property is related to topological stability. In fact, if a dynamical system is topologically stable then it has the inverse shadowing property with respect to the continuous method, but the converse does not hold in general. The following can be found in [12].

Example 1.1 Let $f$ be a diffeomorphism on a manifold $M$. Then we consider the equivalence relation ' $\sim$ ' defined by $(x, 1) \sim(f(x), 0)$ on $M \times[0,1]$, for $x \in M$. Set $M^{\prime}=M \times$ $[0,1] / \sim$. We define a flow $K_{f}$ on $M^{\prime}$ by setting

$$
K_{f}((x, s), t)=\left(f^{[t+s]}(x), t+s-[t+s]\right)
$$

○2014 Lee; licensee Springer. This is an Open Access article distributed under the terms of the Creative Commons Attribution License (http://creativecommons.org/licenses/by/2.0), which permits unrestricted use, distribution, and reproduction in any medium, provided the original work is properly cited. 
for $(x, s) \in M^{\prime}$ and $t \in \mathbb{R}$, where $[t]$ denotes the greatest integer less than or equal to $t$. Then the flow $K_{f}$ on $M^{\prime}$ is called the suspension flow of $f$.

Let $f: S^{1} \rightarrow S^{1}$ be a diffeomorphism such that $f(x)=x+\epsilon x^{4} \sin (\pi / x)$ for $x \neq 0$, and $f(x)=0$ for $x=0$, where $\epsilon>0$ is sufficiently small. Then we know that $f$ has the shadowing property. By Lee and Park [16], it has the inverse shadowing property with respect to the continuous method. But the diffeomorphism is not topologically stable (see [19]). By Thomas [20], the suspension flow $K_{f}$ of $f$ is topologically stable if and only if $f$ is topologically stable. Thus the inverse shadowing is a general notion of topological stability. The notion of the orbital inverse shadowing property was introduced by [12]. It was proved in [12] that if a diffeomorphism belongs to the $C^{1}$-interior of the set of all diffeomorphisms having the orbital inverse shadowing property with respect to the continuous methods, then it is a structurally stable diffeomorphism. For vector fields, Lee et al. [15] proved that if a vector field belongs to the $C^{1}$-interior of the set of all vector fields having the orbital inverse shadowing property with respect to the continuous methods, then it is a structurally stable vector field.

In this paper, we study orbital shadowing and the orbital inverse shadowing property for a Hamiltonian system.

\section{Hamiltonian systems}

Let $(M, \omega)$ be a symplectic manifold, where $M$ is a $2 n(\geq 2)$-dimensional, compact, boundaryless, connected, and smooth Riemannian manifold, endowed with the symplectic form $\omega$. A Hamiltonian $H: M \rightarrow \mathbb{R}$ is a real valued $C^{r}(r \geq 2)$ function on $M$. Denote by $C^{r}(M, \mathbb{R})$ the set of $C^{r}$-Hamiltonian on $M$. In this paper, we consider the $C^{2}$-topology, thus we set $r=2$. Given a Hamiltonian $H$, we define the Hamiltonian vector field $X_{H}$ as follows: for all $v \in T_{p} M$

$$
\omega\left(X_{H}(p), v\right)=d_{p} H(v)
$$

which generates the Hamiltonian flow $X_{H}^{t}$. Note that a Hamiltonian vector field $X_{H}$ is $C^{1}$ if and only if the Hamiltonian function $H$ is $C^{2}$. There exists a formulation in terms of the Hamiltonian equations, that is, the usual Hamiltonian equations are

$$
\dot{q}_{i}=\frac{\partial H}{\partial p_{i}}, \quad \dot{p}_{i}=-\frac{\partial H}{\partial q_{i}},
$$

where $\left(q_{i}, p_{i}\right) \in M$ and $i=1, \ldots, n$. Denote by $\operatorname{Sing}\left(X_{H}\right)$ the set of all singularities of $X_{H}$. Since $H$ is smooth and $M$ is compact, $\operatorname{Sing}\left(X_{H}\right) \neq \emptyset$. A scalar $e \in H(M) \subset \mathbb{R}$ is called the energy of $H$. An energy hypersurface $\mathcal{E}_{H, e}$ is a connected component of $H^{-1}(\{e\})$ called an energy level set. The energy level set $H^{-1}(\{e\})$ is said to be regular if any energy hypersurface of $H^{-1}(\{e\})$ is regular, which means it does not contain singularities. Clearly a regular energy hypersurface is a $X_{H^{t}}^{t}$-invariant, compact, and $(2 n-1)$-dimensional manifold. We say a Hamiltonian level $(H, e)$ is regular if the energy level set $H^{-1}(\{e\})$ is regular. A Hamiltonian system is $\left(H, e, \mathcal{E}_{H, e}\right)$, where $H$ is the Hamiltonian, $e$ is the energy, and $\mathcal{E}_{H, e}$ is a regular connected component of $H^{-1}(\{e\})$. Then $H^{-1}(\{e\})$ corresponds to the union of a finite number of closed connected components, that is, $H^{-1}(\{e\})=\bigcup_{i=1}^{n} \mathcal{E}_{H, e, i}$, for $n \in \mathbb{N}$. Note that Hamiltonian flows are symplectic and volume preserving, which is known as 
the Liouville theorem. Thus the $2 n$-form $\omega^{n}=\omega \wedge \cdots \wedge \omega$ (n-times) is a volume form and induces a measure $\mu$ on $M$, which is called the Lebesgue measure associated to $\omega^{n}$. Then the measure $\mu$ on $M$ is invariant by the Hamiltonian flow. For a regular Hamiltonian level $(H, e)$, we define a volume form $\omega_{\mathcal{E}_{H, e}}$ on each energy hypersurface $\mathcal{E}_{H, e} \subset H^{-1}(\{e\})$, where for all $x \in \mathcal{E}_{H, e}$,

$$
\omega_{\mathcal{E}_{H, e}}(u, v, w)=\omega^{n}\left(\nabla H_{x}, u, v, w\right)
$$

where $\omega_{\mathcal{E}_{H, e}}: T_{x} \mathcal{E}_{H, e} \times T_{x} \mathcal{E}_{H, e} \times T_{x} \mathcal{E}_{H, e} \rightarrow \mathbb{R}$. The volume form $\omega_{H, e}$ is $X_{H}^{t}$-invariant. Since any energy hypersurface is compact, it induces an invariant volume measure $\mu_{\mathcal{E}_{H, e}}$ on $\mathcal{E}_{H, e}$ which is a finite measure. Now we consider the transversal linear Poincaré flow. Given a Hamiltonian vector field $X_{H}$ and a regular point $x \in M$, let $e \in H(x)$. Define $\mathcal{N}_{x}=N_{x} \cap$ $T_{x} H^{-1}(\{e\})$, where $T_{x} H^{-1}(\{e\})=\operatorname{Ker} d H(x)$ is the tangent space to the energy level set. Then $\mathcal{N}_{x}$ is a $(\operatorname{dim} M-2)$-dimensional bundle. The transversal linear Poincaré flow associated to $H, P_{H}^{t}(x): \mathcal{N}_{x} \rightarrow \mathcal{N}_{X_{H}^{t}(x)}$ given by $P_{t}(x)(v)=\Pi_{X_{H}^{t}(x)} \circ D X_{H_{x}^{t}}(v)$, where $\Pi_{X_{H}^{t}(x)}: T_{X_{H}^{t}(x)} M \rightarrow$ $\mathcal{N}_{X_{H}^{t}(x)}$, is the canonical projection. It is clear that $\mathcal{N}_{x}$ is $P_{H}^{t}(x)$-invariant. Let $H \in C^{2}(M, \mathbb{R})$ and let $\Lambda \subset M$ be a $X_{H}^{t}$-invariant, closed, and regular set of $M$. We say that $\Lambda$ is hyperbolic for $P_{H}^{t}$ if $\mathcal{N}_{\Lambda}$ admits a $P_{H}^{t}$-invariant splitting $\Delta_{\Lambda}^{s} \oplus \Delta_{\Lambda}^{u}$ such that for any $\lambda \in(0,1)$ there is $l>0$ such that

$$
\left\|\left.P_{h}^{l}(x)\right|_{\Delta_{x}^{s}}\right\| \leq \lambda \text { and }\left\|\left.P_{H}^{-l}\left(X^{l}(x)\right)\right|_{\Delta_{X^{l}(x)}^{u}}\right\| \leq \lambda
$$

for any $x \in \Lambda$. We say that a Hamiltonian system $\left(H, e, \mathcal{E}_{H, e}\right)$ is Anosov if $\mathcal{E}_{H, e}$ is hyperbolic for the Hamiltonian flow $X_{H}^{t}$ associated to $H$.

\section{Orbital shadowing}

Let $\left(H, e, \mathcal{E}_{H, e}\right)$ be a Hamiltonian system. For any $\delta>0$, a sequence $\left\{\left(x_{i}, t_{i}\right): x_{i} \in \mathcal{E}_{H, e}, t_{i} \geq\right.$ 1 , and $-\infty \leq a<i<b \leq \infty\}$ is a $\delta$-pseudo-orbit of $H$ if $d\left(X_{H}^{t_{i}}\left(x_{i}\right), x_{i+1}\right)<\delta$ for any $a \leq i \leq$ $b-1$. For the sequence $\left\{t_{i}\right\}_{i \in \mathbb{Z}}$, we denote

$$
S_{i}= \begin{cases}t_{0}+t_{1}+\cdots+t_{i-1}, & \text { if } i>0, \\ 0, & \text { if } i=0, \\ -t_{-1}-t_{-2}-\cdots-t_{i}, & \text { if } i<0\end{cases}
$$

We say that $\left(H, e, \mathcal{E}_{H, e}\right)$ has the shadowing property if for any $\epsilon>0$, there is $\delta>0$ that satisfies the following property: given any $\delta$-pseudo-orbit $\left\{\left(x_{i}, t_{i}\right): t_{i} \geq 1, i \in \mathbb{Z}\right\}$ for all $i \in \mathbb{Z}$, there is a point $y \in \mathcal{E}_{H, e}$ and an increasing homeomorphism $h: \mathbb{R} \rightarrow \mathbb{R}$ with $h(0)=0$ such that

$$
d\left(X_{H}^{h(t)}(y), X_{H}^{t-S_{i}}\left(x_{i}\right)\right)<\epsilon
$$

for any $i \in \mathbb{Z}$ and for any $S_{i} \leq t<S_{i+1}$.

Let $A$ and $B$ be closed sets of $M$. Then we can define the Hausdorff distance as follows:

$$
d_{H}(A, B)=\max \left\{\sup _{x \in A} d(x, B), \sup _{y \in B} d(A, y)\right\} .
$$


We introduce the notion of the orbital shadowing property. For $x \in M$, we denote by $\operatorname{Orb}_{X_{H}}(x)$ the orbit of $H$ through $x$; that is, $\operatorname{Orb}_{X_{H}}(x)=\left\{X_{H}^{t}(x): t \in \mathbb{R}\right\}$. We say that $\left(H, e, \mathcal{E}_{H, e}\right)$ has the orbital shadowing property if for any $\epsilon>0$ there is $\delta>0$ such that for any $(\delta, 1)$-pseudo-orbit $\xi=\left\{\left(x_{i}, t_{i}\right): t_{i} \geq 1, i \in \mathbb{Z}\right\}$ there is a point $y \in \mathcal{E}_{H, e}$ such that

$$
d_{H}\left(\overline{\operatorname{Orb}_{X_{H}}(x)}, \bar{\xi}\right)<\epsilon
$$

This means that

$$
\xi \subset B_{\epsilon}\left(\operatorname{Orb}_{X_{H}}(y)\right), \quad \text { and } \quad \operatorname{Orb}_{X_{H}}(y) \subset B_{\epsilon}(\xi)
$$

where $B_{\epsilon}(A)$ is a neighborhood of $A$.

Note that the orbital shadowing property is a weak version of the shadowing property: the difference is that we do not require a point $x_{i}$ of a pseudo-orbit $\xi$ and the point $X_{H}^{t_{i}}(y)$ of an exact orbit $\operatorname{Orb}_{X_{H}}(y)$ to be close 'at any time moment'; instead, the sets of the points of $\xi$ and $\operatorname{Orb}_{X_{H}}(y)$ are required to be close. We say that $\left(H, e, \mathcal{E}_{H, e}\right)$ has the robustly orbitally shadowing property if there is a neighborhood $U$ of $\left(H, e, \mathcal{E}_{H, e}\right)$ such that every $\left(H_{1}, e_{1}, \mathcal{E}_{H_{1}, e_{1}}\right) \in \mathcal{U}$ has the orbital shadowing property.

In [21], Bessa et al. proved that if a Hamiltonian system has the robustly shadowing property then it is Anosov. From this fact, we have the following result, which is more general than the result of [21].

Theorem 3.1 Let $\left(H, e, \mathcal{E}_{H, e}\right)$ be a Hamiltonian system. If $\left(H, e, \mathcal{E}_{H, e}\right)$ has the robustly orbitally shadowing property, then it is Anosov.

\section{Orbital inverse shadowing}

Let $H \in C^{2}(M, \mathbb{R})$, and let $\left(H, e, \mathcal{E}_{H, e}\right)$ be a Hamiltonian system. A mapping $\Psi: \mathbb{R} \times M \rightarrow M$ is called a $(\delta, T)$-method for $H$ if for any $x \in M$, the map $\Psi_{x}: \mathbb{R} \rightarrow M$ defined by

$$
\Psi_{x}(t)=\Psi(t, x), \quad t \in \mathbb{R}
$$

is a $(\delta, T)$-pseudo-orbit of $H . \Psi$ is said to be complete if $\Psi(0, x)=x$ for any $x \in M$. Then a $(\delta, T)$-method for $H$ can be considered as a family of $(\delta, T)$-pseudo-orbit of $H$. A method $\Psi$ for $H$ is said to be continuous if the map $\Psi^{\prime}: M \rightarrow M^{\mathbb{R}}$ given by $\Psi^{\prime}(x)(t)=\Psi(t, x)$ for $x \in M$ and $t \in \mathbb{R}$, is continuous under the compact open topology on $M^{\mathbb{R}}$, where $M^{\mathbb{R}}$ denotes the set of all functions from $\mathbb{R}$ to $M$. The set of all complete $(\delta, 1)$-methods for $H \in C^{2}(M, \mathbb{R})$ will be denoted by $\mathcal{T}_{a}(\delta, H)$. Let $\mathcal{T}_{d}(\delta, H)$ be the set of all complete continuous $(\delta, 1)$-methods for $H$ which are induced by Hamiltonian systems $H_{1}$ with $d_{C^{2}}\left(H, H_{1}\right)<\delta$, where $d_{C^{2}}$ is the $C^{2}$ metric on $C^{2}(M, \mathbb{R})$. We say that a Hamiltonian system $\left(H, e, \mathcal{E}_{H, e}\right)$ has the inverse shadowing property with respect to the class $\mathcal{T}_{d}$ if for any $\epsilon>0$ there is $\delta>0$ such that for any $(\delta, T)$-method $\Psi \in \mathcal{T}_{d}(\delta, H)$ and any point $x \in M$, there are $y \in M$ and an increasing homeomorphism $h: \mathbb{R} \rightarrow \mathbb{R}$ with $h(0)=0$ such that

$$
d\left(X^{h(t)}(x), \Psi(t, y)\right)<\epsilon, \quad t \in \mathbb{R} .
$$

Now we introduce the notion of the orbital inverse shadowing property with respect to the $\mathcal{T}_{d}$. 
We say that $\left(H, e, \mathcal{E}_{H, e}\right)$ has the orbital inverse shadowing property with respect to the class $\mathcal{T}_{d}$ if for any $\epsilon>0$ there is $\delta>0$ such that for any $(\delta, 1)$-method $\Psi \in \mathcal{T}_{d}(\delta, H)$ and any point $x \in M$ there is $y \in M$ such that

$$
d_{H}\left(\overline{\operatorname{Orb}_{X_{H}}(x)}, \overline{\operatorname{Orb}(y, \Psi)}\right)<\epsilon,
$$

where $\operatorname{Orb}(y, \Psi)=\{\Psi(t, y): t \in \mathbb{R}\}$. Note that if $H$ has the inverse shadowing property with respect to the class $\mathcal{T}_{d}$, then $H$ has the orbital shadowing property with respect to the class $\mathcal{T}_{d}$. But the converse is not true. Indeed, an irrational rotation map does not have the inverse shadowing property, and the map has the orbital shadowing property. We say that $\left(H, e, \mathcal{E}_{H, e}\right)$ has the robustly orbitally inverse shadowing property with respect to the class $\mathcal{T}_{d}$ if there is a neighborhood $\mathcal{U}$ of $\left(H, e, \mathcal{E}_{H, e}\right)$ such that for any $\left(H_{1}, e_{1}, \mathcal{E}_{H_{1}, e_{1}}\right) \in \mathcal{U}$ has the orbital inverse shadowing property with respect to the class $\mathcal{T}_{d}$.

In [21], Bessa et al. proved that if a Hamiltonian system is robustly topologically stable then it is Anosov. Note that by the definition of topological stability, the inverse shadowing property with respect to the class of the continuous method is a general notion of topological stability. It means that if a system is topologically stable, then it has the inverse shadowing property with respect to the class of the continuous method, but the converse is not true. From these facts, we have the generalization results as follows.

Theorem 4.1 Let $\left(H, e, \mathcal{E}_{H, e}\right)$ be a Hamiltonian system. If $H$ has the robustly orbitally inverse shadowing property with respect to $\mathcal{T}_{d}$, then it is Anosov.

\section{Proof of Theorem 3.1 and Theorem 4.1}

A Hamiltonian system $\left(H, e, \mathcal{E}_{H, e}\right)$ is a Hamiltonian star system if there is a neighborhood $\mathcal{U}$ of $\left(H, e, \mathcal{E}_{H, e}\right)$ such that for any $\left(H_{1}, e_{1}, \mathcal{E}_{H_{1}, e_{1}}\right) \in \mathcal{U}$, the corresponding regular energy hypersurface $\mathcal{E}_{H_{1}, e_{1}}$ has all hyperbolic closed orbits.

In [22], Bessa et al. showed that if we have a Hamiltonian star system on a four dimensional manifold, then it is Anosov. Afterwards, Bessa et al. proved the following.

Lemma 5.1 [23, Theorem 1] If $\left(H, e, \mathcal{E}_{H, e}\right)$ is a Hamiltonian star system, then it is Anosov.

To prove Theorem 3.1 and Theorem 4.1, it is enough to show that a Hamiltonian system $\left(H, e, \mathcal{E}_{H, e}\right)$ is a Hamiltonian star system. For this, we need the following proposition.

Proposition 5.2 Let $\left(H, e, \mathcal{E}_{H, e}\right)$ be a Hamiltonian system. If the following hold:

(a) $\left(H, e, \mathcal{E}_{H, e}\right)$ has the robustly orbitally shadowing property,

(b) $\left(H, e, \mathcal{E}_{H, e}\right)$ has the robustly orbitally inverse shadowing property with respect to the $\mathcal{T}_{d}$,

then $\left(H, e, \mathcal{E}_{H, e}\right)$ is a Hamiltonian star system.

The next lemma is called a pasting lemma; it was established in [23].

Lemma 5.3 [23, Theorem 5.1] Let $H \in C^{r}(M, \mathbb{R}), 2 \leq r \leq \infty$, and let $K$ be a compact subset of $M$, and $U$ a small neighborhood of $K$. Given $\epsilon>0$ there exists $\delta>0$ such that if $H_{1} \epsilon$ $C^{l}(M, \mathbb{R})$, for $2 \leq l \leq \infty$ is $\delta-C^{\mathrm{min}\{r, l\}}$-close to $H$ on $U$, then there exist $H_{0} \in C^{l}(M, \mathbb{R})$ and a closed set $V$ such that 
(a) $K \subset V \subset U$,

(b) $H_{0}=H_{1}$ on $V$,

(c) $H_{0}=H$ on $U^{c}$,

(d) $H_{0}$ is $\epsilon-C^{\min \{r, l\}}$-close to $H$.

Let $x \in M \backslash \operatorname{Sing}\left(X_{H}\right)$. We define $X_{H}^{\left[t_{1}, t_{2}\right]}(x)=\left\{X_{H}^{t}(x): t \in\left[t_{1}, t_{2}\right]\right\}$. Let $\mathbf{N}_{x}$ be a transversal section to the flow at $x$, a flow box associated to $\mathbf{N}_{x}$ is defined by $\mathcal{F}(x)=X_{H}^{\left[-\tau_{1}, \tau_{2}\right]}\left(\mathbf{N}_{x}\right)$, where $\tau_{1}, \tau_{2}$ are taken small such that $\mathcal{F}(x)$ is a neighborhood of $x$ foliated by regular orbits. The following lemma is a version of Frank's lemma for Hamiltonians.

Lemma 5.4 [24, Theorem 1] Let $H \in C^{r}(M, \mathbb{R}), 2 \leq r \leq \infty, \epsilon>0, \tau>0$ and $x \in M$. Then there is $\delta>0$ such that for any flowbox $\mathcal{F}(x)$ of an injective arc of orbit $X_{H}^{[0, t]}(x)(t \geq \tau)$ and a transversal symplectic $\delta$-perturbation $\Psi$ of $P_{H}^{t}(x)$, there is $H_{0} \in C^{l}(M, \mathbb{R})$ with $l=$ $\max \{2, k-1\}$ such that

(a) $H_{0}$ is $\epsilon-C^{2}$-close to $H$,

(b) $P_{H_{0}}^{t}(x)=\Psi$

(c) $H=H_{0}$ on $X_{H}^{[0, t]}(x) \cup(M \backslash \mathcal{F}(x))$.

Let $\left(H, e, \mathcal{E}_{H, e}\right)$ be a Hamiltonian system and let $p$ be a periodic point in $\mathcal{E}_{H, e}$ with pe$\operatorname{riod} \pi(p)$. For a point $p \in \mathcal{E}_{H, e}, \mathbf{N}_{p} \subset M$ is transverse to the flow, that is, a local $(2 n-1)$ submanifold for which $X_{H}$ is nowhere tangency. Define the $2 n-2$ symplectic submanifold $\mathcal{N}_{p}=\mathbf{N}_{p} \cap \mathcal{E}_{H, e}$. For $x \in \mathcal{N}_{p}, T_{x} \mathcal{E}_{H, e}=T_{x} \mathcal{N}_{p} \oplus\left\langle X_{H}(x)\right\rangle$. Let $U \subset M$ be an open neighborhood of $p$ and $V=U \cap M$. Let $f: V \rightarrow \mathcal{N}_{p}$ be the Poincaré map of $X_{H}^{t}$ to $\mathcal{N}_{p}$ such that $f(x)=X_{H}^{\tau(x)}(x)$ for all $x \in V$, where $\tau(x)$ is the return time to $\mathcal{N}_{p}$ defined by the relation $X_{H}^{\tau(x)}(x) \in \mathcal{N}_{p}$ and $\tau(p)=\pi(p)$. Then $f$ is a $C^{1}$-symplectic diffeomorphism. The following lemma was established in [23, Theorem 5.3].

Lemma 5.5 Let $H \in C^{\infty}(M, \mathbb{R})$ be the Poincaré map $f$ at a periodic point $p$. Then for any $\epsilon>0$ there is $\delta>0$ such that for any symplectic diffeomorphism $g \delta$ - $C^{3}$-close to $f$ there is a Hamiltonian $H_{1} \epsilon-C^{2}$-close with a Poincaré map $g$.

A point $x \in \mathcal{E}_{H, e}$ is a non-wandering point of $H$ if for any neighborhood $U$ of $x$ in $\mathcal{E}_{H, e}$ there is $T>0$ such that $X_{H}^{T}(U) \cap U \neq \emptyset$. Denote by $\Omega\left(\left.H\right|_{\mathcal{E}_{H, e}}\right)$ the set of non-wandering points of $H$ on the energy hypersurface $\mathcal{E}_{H, e}$. We say that $x \in \mathcal{E}_{H, e}$ is a periodic point of $H$ if there is $T>0$ such that $X_{H}^{T}(x)=x$. Denote by $P\left(\left.H\right|_{\mathcal{E}_{H, e}}\right)$ the set of all periodic points of $H$. Given $1 \leq k \leq n-1$, we recall that a $k$-elliptic closed orbit has $2 k$ simple non-real eigenvalues of the transversal linear Poincaré flow at the period of norm 1 , and the norm of its remaining eigenvalues different from 1 . If $k=n-1$ the elliptic closed orbits have all eigenvalues at the period of norm 1 , simple and non-real.

By Abraham's and Marsden's [25] result-the symplectic eigenvalue theorem-if $\lambda$ is an eigenvalue of $D_{p} f^{\pi}$ of multiplicity $k$, then $1 / \lambda$ is an eigenvalue of $D_{p} f^{\pi}$ of multiplicity $k$. Moreover, if the multiplicity of the eigenvalues 1 and -1 , then it is even.

Recall that if we let $\mathcal{W}$ be a small neighborhood of a regular energy hypersurface $\mathcal{E}_{H, e}$, then there exist a small neighborhood $\mathcal{U}$ of the Hamiltonian $H$ and $\epsilon>0$ such that for any $H_{1} \in \mathcal{U}$ and for any $e_{1} \in(e-\epsilon, e+\epsilon)$, we have $H_{1}^{-1}(\{e\}) \cap \mathcal{W}=\mathcal{E}_{H_{1}, e_{1}}$. The energy hypersurface $\mathcal{E}_{H_{1}, e_{1}}$ is called the analytic continuation of $\mathcal{E}_{H, e}$. 
Remark 5.6 By Robinson's version of the Kupka-Smale theorem [26], a $C^{2}$-generic Hamiltonian has all closed orbits of hyperbolic or elliptic type. Thus, we can see that there is a $H_{1}$ close to $H$ such that $H_{1}$ has a $k$-elliptic periodic orbit $p$ of period $\pi(p)$. Therefore, there is a splitting of the normal subbundle $\Delta_{p}^{c}$ along the orbit $p$ into $k$-subspaces such that $\Delta_{p}^{c}=\Delta_{1}^{c} \oplus \cdots \oplus \Delta_{k}^{c}$, where $\operatorname{dim} \Delta_{i}^{c}=2$ for $i=1, \ldots, k$. Let $\lambda_{i}=\exp \left(\theta_{i} j\right)$ is an eigenvalue of $\left.P_{H_{1}}^{\pi(p)}\right|_{\Delta_{i}^{c}}$. If $\theta_{i}$ is an irrational, then, by Frank's lemma, there is $H_{2} C^{2}$-close to $H_{1}$ such that each appropriate restriction of the Poincaré map $\left.f_{H_{2}}\right|_{\mathcal{N}_{i}^{c}}$ is conjugate to a rational rotation, for $i=1, \ldots, k$.

The following is the proof of Proposition 5.2(a).

Lemma 5.7 Let $\left(H, e, \mathcal{E}_{H, e}\right)$ be a Hamiltonian system. If $\left(H, e, \mathcal{E}_{H, e}\right)$ has the robustly orbitally shadowing property then $\left(H, e, \mathcal{E}_{H, e}\right)$ is a Hamiltonian star system.

Proof Let $p \in \gamma \in P\left(\left.H\right|_{\mathcal{E}_{H, e}}\right)$ with $X_{H}^{\pi(p)}(p)=p(\pi(p)>0)$ associated to $\mathcal{E}_{H, e}$. Suppose that $p$ is not hyperbolic for $H$. Then there is an eigenvalue $\lambda$ of $P_{H}^{\pi(p)}(p)$ such that $|\lambda|=1$. Then by Lemma 5.3 and Lemma 5.4, we can find $H_{1} C^{2}$-close to $H$ such that $H_{1}$ has a nonhyperbolic periodic point $p_{1}$ close to $p$ with period $\pi\left(p_{1}\right)$ close to $\pi(p)$. It is clear that $p_{1}$ is not the analytic continuation to $p$. Then as in the proof of the [27, Theorem 4.3], we can make the Poincaré map $f$ at $p_{1}$ associated to the Hamiltonian flow $X_{H_{1}}^{t}$ a $C^{\infty}$ local symplectic diffeomorphism, that is, $f: \mathcal{N}_{p_{1}, r} \rightarrow \mathcal{N}_{p_{1}}$ for some $r>0$. For simplicity, we assume that $p_{1}$ is 1-elliptic. Then $T_{p_{1}} \mathcal{N}_{p_{1}, r}=\Delta_{p_{1}}^{c} \oplus \Delta_{p_{1}}^{s} \oplus \Delta_{p_{1}}^{u}$, where $\Delta_{p_{1}}^{c}$ is associated to $\lambda, \Delta_{p_{1}}^{c}$ is associated to an eigenvalues less than 1 and $\Delta_{p_{1}}^{u}$ associated to an eigenvalue greater than 1 . By Lemma 5.4, Lemma 5.5 and the Darboux theorem, there are $\epsilon_{0}>0$ and a linear map $A: N_{p_{1}} \rightarrow N_{p_{1}}$ such that $g(x)=\varphi_{p_{1}} \circ A \circ \varphi_{p_{1}}^{-1}(x)$ for $x \in B_{\epsilon_{0}}\left(p_{1}\right) \cap \mathcal{N}_{p_{1}, r}$ and $g(x)=f(x)$ for $x \notin B_{\epsilon_{0}}\left(p_{1}\right) \cap \mathcal{N}_{p_{1}, r}$, where $\varphi_{p_{1}}: B_{\epsilon_{0}}\left(p_{1}\right) \cap \mathcal{N}_{p_{1}, r} \rightarrow T_{p_{1}} \mathcal{N}_{p_{1}}$ with $\varphi_{p_{1}}\left(p_{1}\right)=\overrightarrow{0}$. It is clear that $g: \mathcal{N}_{p_{1}, r} \rightarrow \mathcal{N}_{p_{1}}$ is the Poincaré map $H_{1}$. Using Lemma 5.4, $\lambda$ can be taken as a rational rotation, that is, there is $l>0$ such that for any $x \in \varphi_{p_{1}}^{-1}\left(\Delta_{p_{1}}^{c}\right) \cap B_{\epsilon_{0}}\left(p_{1}\right) \subset \mathcal{N}_{p_{1}, r}$, we have $g^{l}(x)=x$. Put $\mathcal{N}_{p_{1}}^{c}=\varphi_{p_{1}}^{-1}\left(\Delta_{p_{1}}^{c}\right) \cap B_{\epsilon_{0}}\left(p_{1}\right)$. For simplicity, we may assume that $g^{l}=g$. Then $x$ is a fixed point for $g$, and the orbit of $x$ is periodic, that is, $X_{H_{1}}^{\pi(x)}(x)=x$, where $\pi(x)$ is the period of $x$. Let $x_{0}=p_{1}$. Take $\epsilon=\min \left\{\epsilon_{0} / 16, r / 8\right\}$, and let $0<\delta<\epsilon$ be the number of the orbital shadowing property. Take $y \in \mathcal{N}_{p_{1}}^{c}$ such that $d\left(p_{1}, y\right)=2 \epsilon$. Now, we construct a $\delta$-pseudo-orbit $\left\{\left(x_{i}, t_{i}\right): t_{i}>0, i \in \mathbb{Z}\right\} \subset \mathcal{N}_{p_{1}}^{c}$ as follows: (i) $t_{i}=\pi\left(p_{1}\right)$ for all $i \in \mathbb{Z}$, and $x_{n}=y$ for some $n \in \mathbb{N}$, (ii) $x_{i}=p_{1}$ for $i \leq 0$, (iii) $d\left(g\left(x_{i}\right), x_{i+1}\right)<\delta$ for $1 \leq i \leq n-1$, and (iv) $x_{i}=y$ for $i \geq n$. By the orbital shadowing property, there is $z \in \mathcal{E}_{H_{1}, e_{1}}$ such that

$$
d_{H}\left(\overline{\operatorname{Orb}_{X_{H_{1}}}(z)}, \overline{\left\{\left(x_{i}, t_{i}\right): t_{i}=\pi\left(p_{1}\right), i \in \mathbb{Z}\right\}}\right)<\epsilon .
$$

If $z \in \mathcal{E}_{H_{1}, e_{1}} \backslash \mathcal{N}_{p_{1}}^{c}$, then, by the hyperbolicity, there is $k \in \mathbb{Z}$ such that

$$
d\left(X_{H_{1}}^{k \pi\left(p_{1}\right)}(z), \overline{\left\{\left(x_{i}, t_{i}\right): t_{i}=\pi\left(p_{1}\right), i \in \mathbb{Z}\right\}}\right)>\epsilon .
$$

This is a contradiction. Thus the orbital shadowing point $z \in \mathcal{N}_{p_{1}}^{c}$. Since $g(z)=z$, we have $X_{H_{1}}^{j \pi\left(p_{1}\right)}(z)=z$ for all $j \in \mathbb{Z}$. By the orbital shadowing property, if there is $x_{k} \in\left\{\left(x_{i}, t_{i}\right): t_{i}=\right.$ $\left.\pi\left(p_{1}\right), i \in \mathbb{Z}\right\}$ such that $d\left(x_{k}, z\right)<\epsilon$, then we can see that there is $x_{l} \in\left\{\left(x_{i}, t_{i}\right): t_{i}=\pi\left(p_{1}\right), i \in\right.$ 
$\mathbb{Z}$ \} such that

$$
d\left(x_{l}, X_{H_{1}}^{j \pi\left(p_{1}\right)}(z)\right)=d\left(x_{l}, z\right)>\epsilon,
$$

for some $j \in \mathbb{Z}$. Thus $\left\{X_{H_{1}}^{t}(z): t \in \mathbb{R}\right\} \not \subset B_{\epsilon}\left(\left\{\left(x_{i}, t_{i}\right): t_{i}=\pi\left(p_{1}\right), i \in \mathbb{Z}\right\}\right)$, which is a contradiction.

Let $p \in H$ be a non-hyperbolic periodic point. Then there is a Hamiltonian $H_{1} C^{2}$-close to $H$ such that $H_{1}$ has a continuation of periodic points close to $p$. For a Hamiltonian system $\left(H, e, \mathcal{E}_{H, e}\right)$ and a periodic point $p \in \mathcal{E}_{H, e}$ with period $\pi(p)$, let $\mathcal{N}_{p}^{c}$ be a submanifold $\mathcal{N}_{p}$ associated to $p$. Then we have the following.

Lemma 5.8 [21, Lemma 6.1] Let $\left(H, e, \mathcal{E}_{H, e}\right)$ be a Hamiltonian system, and let $p \in \mathcal{E}_{H, e}$ be a non-hyperbolic periodic point. Then there is a Hamiltonian system $\left(H_{1}, e_{1}, \mathcal{E}_{H_{1}, e_{1}}\right) C^{2}$-close to $\left(H, e, \mathcal{E}_{H, e}\right)$ such that $H_{1}$ has a non-hyperbolic periodic point $q \in \mathcal{E}_{H_{1}, e_{1}}$ close to $p$ and every point in a small neighborhood of $q \in \mathcal{N}_{p}^{c}$ is a periodic point of $H_{1}$.

The following is the proof of Proposition 5.2(b).

Lemma 5.9 Let $\left(H, e, \mathcal{E}_{H, e}\right)$ be a Hamiltonian system. If $\left(H, e, \mathcal{E}_{H, e}\right)$ has the robustly orbitally inverse shadowing property with respect to the class $\mathcal{T}_{d}$, then $\left(H, e, \mathcal{E}_{H, e}\right)$ is a Hamiltonian star system.

Proof Let $\mathcal{U} \subset C^{2}(H, \mathbb{R})$ be a $C^{1}$-neighborhood of $H$. Suppose that $p \in \mathcal{E}_{H, e}$ is not a hyperbolic periodic point. By Lemma 5.8, there are $H_{1} \in \mathcal{U}$ and $\eta>0$ such that (i) $H_{1}$ has a non-hyperbolic periodic point $q \in \mathcal{E}_{H_{1}, e_{1}}$, and (ii) every $\gamma \in B_{\eta}(q) \cap \mathcal{N}_{q}^{c}$ is a periodic point of $H_{1}$. Take $0<\epsilon<\eta / 16$, and let $0<\delta<\epsilon$ be as in the definition of the orbital inverse shadowing property of $H_{1}$. Let $f_{H_{1}}$ be the Poincaré map at $q$ associated to $P_{H_{1}}^{t}$. Since every $\gamma \in B_{\eta}(q) \cap \mathcal{N}_{q}^{c}$ is a periodic point of $H_{1}$, we know that $f_{H_{1}}(\gamma)=\gamma$.

Take $0<\tau<1$ such that it is sufficiently small. Then we define the Poincaré map $g_{H_{1}}$ near $q$ by $g_{H_{1}}(x)=\left(\tau x_{1}, x^{\prime}\right)$ for $x \in B_{\eta}(q) \cap \mathcal{N}_{q}^{c}$ such that $d_{C^{3}}\left(f_{H_{1}}, g_{H_{1}}\right)<\delta$, where $x_{1}$ is the 1-component of $x$ and $x^{\prime}$ is the other component. By Lemma 5.5, there is a Hamiltonian $H_{1}$ which is associated to the Poincaré map $g_{H_{1}}$, and $d_{C^{2}}\left(H_{1}, H_{2}\right)<\delta$. Take $y \in B_{\eta}(q) \cap \mathcal{N}_{q}^{c}$ such that $d(y, q)=2 \epsilon$. Then for any $z \in \mathcal{E}_{H_{1}, e_{1}}$,

$$
d_{H}\left(\overline{\operatorname{Orb}_{X_{H_{1}}}(y)}, \overline{\operatorname{Orb}_{X_{H_{2}}}(z)}\right)<\epsilon .
$$

If $y=z$ then $f_{H_{1}}(y)=y$ and $g_{H_{1}}^{i}(y)=\left(\tau^{i} y_{1}, y^{\prime}\right)$ for all $i \in \mathbb{Z}$. Then there is $k \in \mathbb{Z}$ such that for $\left.g_{H_{1}}\right|_{\mathcal{N}_{q}^{c}}$,

$$
g_{H_{1}}^{k}(y)=\left(\tau^{k} y_{1}, y^{\prime}\right) \notin B_{\epsilon}\left(\left\{f_{H_{1}}^{i}(y): i \in \mathbb{Z}\right\}\right) .
$$

Then we know that $d_{H}\left(\overline{\operatorname{Orb}_{X_{H_{1}}}(y)}, \overline{\operatorname{Orb}_{X_{H_{2}}}(z)}\right)>\epsilon$. This is a contradiction, since $H_{1}$ has the orbital inverse shadowing property.

Finally we consider $y \neq z$. Set $z^{\prime}=X_{H_{1}}^{t^{\prime}}(z) \in B_{\eta}(q) \cap \mathcal{N}_{q}^{c}$, where $\left|t^{\prime}\right|=\min \left\{|t|: X_{H_{1}}^{t}(z) \in\right.$ $\left.B_{\eta}(q) \cap \mathcal{N}_{q}^{c}\right\}$. Since

$$
d_{H}\left(\overline{\operatorname{Orb}_{X_{H_{1}}}(y)}, \overline{\operatorname{Orb}_{X_{H_{2}}}(z)}\right)<\epsilon,
$$


we have $d\left(X_{H_{1}}^{t}(y), g_{H_{1}}^{i}\left(z^{\prime}\right)\right)<\epsilon$ for some $t \in \mathbb{R}$. Since $d_{C^{2}}\left(H_{1}, H_{2}\right)<\delta$, we can choose a sequence $\left\{g_{H_{1}}^{i}\left(z^{\prime}\right)\right\} \subset B_{\epsilon}(q) \cap \mathcal{N}_{q}^{c}$ such that $d\left(f_{H_{1}}^{i}(y), g_{H_{1}}^{i}(z)\right)<\epsilon$ for all $i \in \mathbb{Z}$. By hyperbolicity, there is $k>0$ such that

$$
g_{H_{1}}^{k}(z) \notin B_{\epsilon}\left(\left\{f_{H_{1}}^{i}(y): i \in \mathbb{Z}\right\}\right)
$$

Thus $B_{\epsilon}\left(\left\{g_{H_{1}}^{i}(z): i \in \mathbb{Z}\right\}\right) \not \subset B_{\epsilon}(q) \cap \mathcal{N}_{q}^{c}$. This is a contradiction by the orbital inverse shadowing property.

\section{Competing interests}

The author declares that they have no competing interests.

\section{Acknowledgements}

This work is supported by Basic Science Research Program through the National Research Foundation of Korea (NRF) funded by the Ministry of Science, ICT \& Future Planning (No-2014R1A1A1A05002124).

Received: 26 February 2014 Accepted: 16 June 2014 Published: 23 Jul 2014

\section{References}

1. Sakai, K: Pseudo-orbit tracing property and strong transversality of diffeomorphisms on closed manifolds. Osaka J. Math. 31, 373-386 (1994)

2. Pilygin, SY, Rodionova, AA, Sakai, K: Orbital and weak shadowing properties. Discrete Contin. Dyn. Syst. 9, 287-303 (2003)

3. Lee, K, Lee, M: Symplectic diffeomorphisms with orbital shadowing. J. Chungcheong Math. Soc. 25, $739-745$ (2012)

4. Lee, K, Lee, M: Volume-preserving diffeomorphisms with orbital shadowing. J. Inequal. Appl. 2013, Article ID 18 (2013)

5. Lee, K, Lee, M: Generic diffeomorphisms with orbital shadowing. Preprint

6. Lee, M: Robustly chain transitive sets with orbital shadowing diffeomorphisms. Dyn. Syst. 27, 507-514 (2012)

7. Lee, M: Volume-preserving diffeomorphisms with inverse shadowing. J. Inequal. Appl. 2012, Article ID 275 (2012)

8. Lee, M: Symplectic diffeomorphisms with inverse shadowing. J. Inequal. Appl. 2013, Article ID 74 (2013)

9. Corless, R, Pilyugin, S: Approximate and real trajectories for generic dynamical systems. J. Math. Anal. Appl. 189, 409-423 (1995)

10. Pilyugin, S: Inverse shadowing by continuous methods. Discrete Contin. Dyn. Syst. 8, 29-38 (2002)

11. Lee, K: Continuous inverse shadowing and hyperbolicity. Bull. Aust. Math. Soc. 67, 352-359 (2004)

12. Choi, T, Lee, $K$, Zhang, Y: Characterisations of $\Omega$-stability and structurally stability via inverse shadowing. Bull. Aust. Math. Soc. 74, 185-196 (2006)

13. Han, Y, Lee, K: Inverse shadowing for structurally stable flows. Dyn. Syst. 19, 371-388 (2004)

14. Lee, K, Lee, M: Stably inverse shadowable transitive sets and dominated splitting. Proc. Am. Math. Soc. 140, 217-226 (2012)

15. Lee, K, Lee, Z, Zhang, Y: Structural stability vector fields with orbital inverse shadowing. J. Korean Math. Soc. 45, 1505-1521 (2008)

16. Lee, K, Park, J: Inverse shadowing of circle maps. Bull. Aust. Math. Soc. 69, 353-359 (2004)

17. Lee, M: Orbital shadowing property for generic divergence-free vector fields. Chaos Solitons Fractals 54, 71-75 (2013)

18. Lee, M: Orbital shadowing for $C^{1}$-generic volume preserving diffeomorphisms. Abstr. Appl. Anal. 2013, Article ID 693032 (2013)

19. Yano, K: Topological stable homeomorphisms of the circle. Nagoya Math. J. 79, 145-149 (1980)

20. Thomas, R: Topological stability: some fundamental properties. J. Differ. Equ. 59, 103-122 (1985)

21. Bessa, M, Rocha, J, Torres, M: Shades of hyperbolicity for Hamiltonians. Nonlinearity 26, 2851-2873 (2013)

22. Bessa, M, Ferreira, C, Rocha, J: On the stability of the set of hyperbolic closed orbits of a Hamiltonian. Math. Proc. Camb. Philos. Soc. 149, 373-383 (2010)

23. Bessa, M, Rocha, J, Torres, M: Hyperbolicity and stability for Hamiltonian flows. J. Differ. Equ. 254, $309-322$ (2013)

24. Vivier, T: Robustly transitive 3-dimensional regular energy surfaces are Anosov. Institut de Math. de Bourgogne, Dijon. Preprint 412 (2005)

25. Abraham, R, Marsden, JE: Foundations of Mechanics, 2nd edn. Benjamin-Cummings, New York (1980)

26. Robinson, C: Generic properties of conservative systems. Am. J. Math. 92, 747-817 (1970)

27. Arbieto, A, Senos, L, Sodera, T: The specification property for flows from the robust and generic view point. J. Differ. Equ. 253, 1893-1909 (2012)

10.1186/1687-1847-2014-192

Cite this article as: Lee: Hamiltonian systems with orbital, orbital inverse shadowing. Advances in Difference Equations 2014, 2014:192 